\title{
A solution to inappropriate self-citation via peer review
}

$\mathrm{O}$ n two occasions recently, peer reviewers of manuscripts we submitted for possible publication recommended manuscript revisions that included citations to minor, tangentially related articles, including some of poor quality. In both cases, the recommended citations were all for publications from a single research group.

Some authors inflate their citation counts through self-citation ${ }^{1}$ and some journal editors ask authors to cite articles from their journal to increase the journal's impact factor. ${ }^{2}$ There is even a well-documented case of Cyril Burt, who edited the British Journal of Statistical Psychology from 1947 to 1963, inserting citations of his work into others' articles without permission. ${ }^{3}$

We know of only one study related to inappropriate peer reviewer requests for citation of their own work. In that study, ${ }^{4} 23 \%$ of 283 researchers indicated that a reviewer had "required them to include unnecessary references to his/her publication(s)." Editors in some cases identify inappropriate reviewer self-citations and remove them before forwarding reviews to authors. Nonetheless, based on the limited evidence available, our experiences and comments of others, ${ }^{5}$ it appears that attempts at self-citation via peer review are not uncommon.

As with author and journal self-citation, self-citation via the peer review process confers an unfair advantage by distorting citation metrics. ${ }^{1-2}$ Requesting citation of one's own work via peer review is of potentially even greater concern, however, because peer reviewers have an important influence on whether a manuscript will be published. Reviewer comments intended primarily to encourage citation of a reviewer's own work are in conflict with the two roles of the peer reviewer: to help the editor determine a manuscript's merit and to help authors produce the most scientifically rigorous report possible. Furthermore, authors who receive inappropriate self-citation

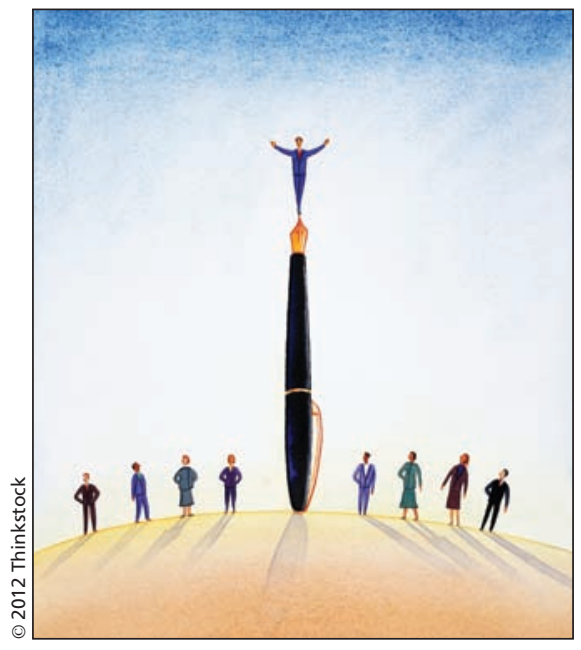

requests from a reviewer are put in the position of having to decide whether to alter their manuscript, possibly changing content substantively, or to argue against incorporating reviewer recommendations, which may raise concerns about whether their manuscript will be accepted for publication.

Journal editors are best positioned to address this problem and, in an ideal world, would easily recognize and remove unnecessary self-citations from reviewer comments. Recognizing unnecessary self-citation by reviewers isn't always obvious, however. Editors are often not content experts on the manuscripts under review. Furthermore, recommended citations often don't mention all the authors.

Open publication of peer reviews, including reviewer names, for both accepted and rejected articles, could discourage peer reviewers from making frivolous recommendations for citation of their work. ${ }^{1}$ However, few journals make reviews of even accepted articles available to readers. ${ }^{6}$ Open peer review, in which reviewers must identify themselves to authors, would also presumably discourage abuses and, in cases where it did not, would allow authors to more easily point out abuses to editors.

Most medical journals, however, including CMAJ, use anonymous peer review. Reviewer guidelines often require that peer reviewers declare relationships that could be perceived as potential conflicts of interests (COIs). Defined by the Institute of Medicine, "A conflict of interest is a set of circumstances that creates a risk that professional judgment or actions regarding a primary interest will be unduly influenced by a secondary interest." COI is present whenever a peer reviewer, appropriately or inappropriately, recommends that authors cite his or her own work. Because peer reviewers must already declare COIs, such as financial interests, it would be relatively simple to require reviewers to declare potential COIs resulting from suggestions to cite their own work. Reviewer instructions could include a statement, such as, "We discourage reviewers from recommending citation of their own work when not clearly necessary to improve the quality of the manuscript under review. Please state in your comments to the editor if you have recommended citation of your own work and the reason for this recommendation."

Many recommendations by reviewers to cite their own work are appropriate and reflect the reviewer's expertise on a manuscript topic. These would not be affected by disclosure. Disclosure would, on the other hand, be an effective deterrent to reviewers who might otherwise make inappropriate requests for citation of one or more of their own articles and would help editors to more easily recognize abuses that are not readily identified otherwise.

\section{Brett D. Thombs PhD \\ Senior Investigator \\ Lady Davis Institute for Medical Research Jewish General Hospital \\ Ilya Razykov BA \\ Master's student \\ McGill University \\ Montréal, Que.}

For references, see Appendix 1, available at www.cmaj.ca/lookup/suppl/doi:10.1503 /cmaj.120597/-/DC1

CMAJ 2012. DOI:10.1503/cmaj.120597

All editorial matter in CMAJ represents the opinions of the authors and not necessarily those of the Canadian Medical Association. 